\title{
Calogero-Moser hierarchy and KP hierarchy
}

\author{
Takahiro Shiota \\ Department of Mathematics, Kyoto University \\ Kyoto 606-01, JAPAN
}

\section{Introduction}

In [1], Airault, McKean and Moser observed that the motion of poles of a rational solution to the K-dV or Boussinesq equation obeys the Calogero-Moser dynamical system [2, 3, 4] with an extra condition on the configuration of poles. In [8], Krichever observed that the motion of poles of a solution to the KP equation which is rational in $t_{1}$ obeys the Calogero-Moser dynamical system. In this note we shall generalize those results to the KP hierarchy. Noting that a pole of a KP solution comes from a zero of the corresponding $\tau$-function, the statement becomes thus

Theorem. (1) Let $\tau\left(t_{1}, t_{2}, t_{3}\right)$ be a monic polynomial in $t_{1}$ whose coefficients depend on $t_{2}$ and $t_{3}$. If $\tau$ satisfies the KP equation

$$
\left(D_{1}^{4}+3 D_{2}^{2}-4 D_{1} D_{3}\right) \tau \cdot \tau=0,
$$

then $\tau$ can be extended uniquely to a $\tau$-function of the KP hierarchy which is also a monic polynomial in $t_{1}$.

(2) Let $\tau=\tau(t)$, where $t=\left(t_{1}, t_{2}, \ldots\right)$, be a monic polynomial in $t_{1}$ whose coefficients depend on $t^{\prime}:=\left(t_{2}, t_{3}, \ldots\right)$. Then $\tau$ is a $\tau$-function of the KP hierarchy if and only if the motion of zeros of $\tau$ is governed by the hierarchy of Calogero-Moser dynamical systems. Namely, writing

$$
\tau(t)=\prod_{i=1}^{N}\left(t_{1}-x_{i}\left(t^{\prime}\right)\right)
$$

locally in $t^{\prime}, \tau$ is a $\tau$-function if and only if

$$
\frac{\partial}{\partial t_{n}}\left(\begin{array}{c}
x_{i} \\
\xi_{i}
\end{array}\right)=(-1)^{n}\left(\begin{array}{r}
\partial H_{n} / \partial \xi_{i} \\
-\partial H_{n} / \partial x_{i}
\end{array}\right), \quad n=2,3, \ldots,
$$

where $\xi_{i}=(1 / 2) \partial x_{i} / \partial t_{2}, H_{n}=\operatorname{tr} Y^{n}$, and $Y$ is the Moser matrix, i.e., $Y=Y\left(t^{\prime}\right)$ is an $N \times N$ matrix such that

$$
Y_{i j}=\frac{1}{x_{i}-x_{j}} \quad(i \neq j), \quad Y_{i i}=\xi_{i}
$$


Note that $x_{i}\left(t^{\prime}\right) \neq x_{j}\left(t^{\prime}\right)$ for $i \neq j$ and almost every $t^{\prime}$ since the zeros of $\tau$ as a function of $t_{1}$ is generically simple (see, e.g., [10, Prop. 8.6]).

This class of KP solutions (let us call them quasi-rational) might not have drawn so much attention as the rational solutions, the ones whose $\tau$-functions are polynomials in all time variables. Rational solutions have been studied in detail as they play the central role in the Kyoto school's theory of soliton equations. Recent developments in the study of the Calogero-Moser hierarchy may lead to a better understanding of quasi-rational solutions.

Rational solutions are clearly円 quasi-rational, but the converse is not necessarily true. The difference between the rational and quasi-rational solutions, and other properties of quasi-rational solutions, can be seen easily from the following very explicit formula:

Corollary 1. Let $\tau$ be the $\tau$-function of a quasi-rational solution of the KP hierarchy (i.e., $\tau$ is a monic polynomial in $t_{1}$ with variable coefficients). Using the notation as in part (2) of the theorem, and assuming for simplicity that $x_{i}(0) \neq$ $x_{j}(0)$ for $i \neq j$, we have

$$
\tau(t)=\operatorname{det}\left(-X_{0}+\sum_{n=1}^{\infty}(-1)^{n-1} n t_{n}\left(Y_{0}\right)^{n-1}\right),
$$

where $X_{0}=\operatorname{diag}\left(x_{1}(0), \ldots, x_{N}(0)\right)$, and $Y_{0}$ is the Moser matrix at $t^{\prime}=0$, i.e.,

$$
\left(Y_{0}\right)_{i j}=\frac{1}{x_{i}(0)-x_{j}(0)} \quad(i \neq j), \quad Y_{i i}=\xi_{i}(0) .
$$

This follows immediately from part (2) of the theorem and a well-known formula on the Calogero-Moser hierarchy. It implies, for example, that a quasirational solution is a rational solution if and only if $Y_{0}$ is nilpotent, and that the $\tau$-function of a quasi-rational solution is actually a polynomial in any first finitely many time variables. Hence we have

Corollary 2. A solution to the KP hierarchy is quasi-rational if and only if it is a finite dimensional solution [7, 9, 10, 11] whose spectral curve $C$ is a rational curve (i.e., its normalization is the Riemann sphere) with only cusp-like singularities (possibly including higher cusps).

Indeed, from (5) the orbit of a quasi-rational solution is a finite dimensional affine space. Since the orbit is isomorphic to the (generalized) Jacobian of $C, C$ is a rational curve with only cusp-like singularities. Conversely, the $\tau$-function belonging to a rational spectral curve with cusp-like singularities gives a polynomial on the Jacobian as a regular function on the affine space, proving the corollary.

\footnotetext{
${ }^{1}$ For a $\tau$-function which is a polynomial in $t_{1}$, it is easy to observe that the coefficient of the top degree term in $t_{1}$ does not vanish. In particular, the coefficient is a constant if $\tau$ is a polynomial in all variables.
} 
A closer look reveals that the singularities of $C$ are at the zeros of $\operatorname{det}\left(z+Y_{0}\right)$, i.e., the negative of the eigenvalues of $Y_{0}$, where $z$ is the global coordinate on the normalization of $C$ given by the analytic extension of the formal spectral parameter in the Baker-Akhiezer function (7).

There should be no essential difficulties in generalizing our theorem to the trigonometric or elliptic Calogero-Moser hyerarchy, except that in the elliptic case the arguments relying on the vanishing of logarithmic derivatives of $\tau$ at $t_{1}=\infty$ no longer work. The trigonometric or elliptic analogue of corollary 2, e.g., to study the geometry of spectral curves which give KP solutions periodic in $t_{1}$, may be less trivial. A. Treibich and J.-L. Verdier have some results in this direction [12]. After completion of this work, the author found out that Alex Kasman [6] has similar work.

\section{Proof of theorem}

Throughout, we use the standard notation for the KP hierarchy. We shall denote $\partial_{x}=\partial / \partial x$ and $\partial_{i}=\partial / \partial t_{i}$. By abuse of notation, we shall "identify" $t_{1}$ and $x$, and simply denote $t_{1}+x$ by $t_{1}$. Our proof is similar to the extension procedure of 11 , Sect. 3]. Starting from Krichever's polynomial solution of the KP equation [8], we shall construct the Baker-Akhiezer function in such a way that the resulting extension of $\tau$ remains to be a polynomial in $t_{1}$. Then we shall observe that the time evolution of zeros is governed by the Calogero-Moser hierarchy.

First assume $\tau=\tau\left(t_{1}, t_{2}, t_{3}\right)$ is of the form (2), except that $t^{\prime}$ is replaced by $\left(t_{2}, t_{3}\right)$, and satisfies the KP equation. Krichever [8] observed that $x_{i}$ and $\xi_{i}=(1 / 2) \partial_{2} x_{i}$ satisfy the equations (3) for $n=2$, 3. In particular, we have

$$
\partial_{2} \xi_{i}=-\frac{\partial}{\partial x_{i}} \operatorname{tr} Y^{2}=-4 \sum_{j(\neq i)} \frac{1}{\left(x_{i}-x_{j}\right)^{3}} .
$$

To extend $\tau$ to a $\tau$-function of the whole KP hierarchy, we construct the BakerAkhiezer (wave) function

$$
w=\left(\sum_{m=0}^{\infty} w_{m} z^{-m}\right) e^{\sum t_{i} z^{i}}
$$

where $w_{m}=w_{m}(t)$ and $w_{0} \equiv 1$. Since $\tau$ is of the form (2), we have

$$
u_{2}:=\partial_{1}^{2} \log \tau=\sum_{i=1}^{N} \frac{-1}{\left(t_{1}-x_{i}\right)^{2}}
$$

so that the equation $\partial_{2} w=B_{2} w, B_{2}:=\partial_{x}^{2}+2 u_{2}$, becomes

$$
\partial_{1} w_{m+1}=\frac{1}{2}\left(\partial_{2}-\partial_{x}^{2}\right) w_{m}+\sum_{i=1}^{N} \frac{1}{\left(t_{1}-x_{i}\right)^{2}} w_{m}, \quad m=0,1, \ldots
$$


Since after the desired extension we should have

$$
w_{m}=\frac{p_{m}(-\tilde{\partial}) \tau}{\tau}, \quad m \geq 0
$$

(see [5]), $w_{m}, m>0$, should have at most simple poles along the zero locus of $\tau$, and vanishes at infinity:

$$
\left.w_{m+1}\right|_{t_{1}=\infty}=0, \quad m=0,1, \ldots
$$

Given $w_{m}$, (8) and (9) determine $w_{m+1}$ uniquely. We shall show that for $m \geq 0$, $w_{m+1}$ has at most simple poles, i.e., it has the form

$$
w_{m+1}=\sum_{i=1}^{N} \frac{c_{m+1, i}}{t_{1}-x_{i}}, \quad m \geq 0 .
$$

For $m=0$, the right hand side of (8) becomes $\sum_{i} 1 /\left(t_{1}-x_{i}\right)^{2}$ since $w_{0} \equiv 1$. Hence

$$
c_{1, i}=-1 \text {. }
$$

For $m>0$, the coefficient of $\left(t_{1}-x_{i}\right)^{-2}$ in (8) gives the recurrence relation

$$
\begin{aligned}
-c_{m+1, i} & =\frac{1}{2}\left(\partial_{2} x_{i}\right) c_{m, i}+\sum_{j(\neq i)} \frac{c_{m, j}}{x_{i}-x_{j}} \\
& =\xi_{i} c_{m, i}+\sum_{j(\neq i)} \frac{c_{m, j}}{x_{i}-x_{j}}
\end{aligned}
$$

and the coefficient of $\left(t_{1}-x_{i}\right)^{-1}$ in $(\mathbb{8})$ gives the constraint

$$
0=\frac{1}{2} \partial_{2} c_{m, i}+\sum_{j(\neq i)} \frac{c_{m, i}-c_{m, j}}{\left(x_{i}-x_{j}\right)^{2}} .
$$

Let $\mathbf{c}_{m}={ }^{t}\left(c_{m, 1}, \ldots, c_{m, N}\right)$, and $\overrightarrow{\mathbf{1}}={ }^{t}(1, \ldots, 1)$. From (11) and (12) we have

$$
\mathbf{c}_{m}=(-1)^{m} Y^{m-1} \overrightarrow{\mathbf{1}},
$$

where $Y$ is the Moser matrix as in (4). The condition (13) is automatically satisfied. This follows from the local regularity of a $\tau$-function [11, p. 366, lemma 8] (since otherwise $w_{m+1}$, and hence the extended $\tau$, would be multivalued), but in the present case we have a more direct proof: From (4), (6) and the definition of $\xi_{i}$, we have the well-known relation

$$
\partial_{2} Y=2[Z, Y], \quad \text { where } \quad Z_{i j}= \begin{cases}1 /\left(x_{i}-x_{j}\right)^{2} & (i \neq j) \\ -\sum_{k(\neq i)} 1 /\left(x_{i}-x_{k}\right)^{2} & (i=j)\end{cases}
$$


Applying this to (14), and noting $Z \overrightarrow{\mathbf{1}}=0$, we have

$$
\frac{1}{2} \partial_{2} \mathbf{c}_{m}=\frac{(-1)^{m}}{2} \partial_{2}\left(Y^{m-1}\right) \overrightarrow{\mathbf{1}}=(-1)^{m}\left[Z, Y^{m-1}\right] \overrightarrow{\mathbf{1}}=(-1)^{m} Z Y^{m-1} \overrightarrow{\mathbf{1}}=Z \mathbf{c}_{m},
$$

which is equivalent to (13).

Now we need a little formal nonsense. We extend $w$ formally to the whole KP hierarchy by solving the equations

$$
\partial_{n} w=\left(W \partial_{x}^{n} W^{-1}\right)_{+} w
$$

for $n=3,4, \ldots$, where $W=\sum_{m=0}^{\infty} w_{m} \partial_{x}^{-m}$. Since those equations are compatible with one another, $w$ extends uniquely to the Baker-Akhiezer function of the whole KP hierarchy. We shall denote the extended $w, w_{m}$ and $\tau$ by $\bar{w}, \bar{w}_{m}$ and $\bar{\tau}$, respectively. From (10) and (16), $\bar{w}_{m}$ are also single valued, and vanish at $t_{1}=\infty$. Hence, since $-\partial_{1} \log \bar{\tau}=\bar{w}_{1}=O\left(z^{-1}\right), \bar{\tau}$ has a polynomial growth, so that it has a pole at $t_{1}=\infty$. Also, each zero of $\tau$ extends to a zero of $\bar{\tau}$ (see [11, lemma 8]). Hence $\bar{\tau}$ is a polynomial in $t_{1}$. Since

$$
-(1 / m) \partial_{m} \log \bar{\tau}+\frac{P\left(\partial_{1}, \ldots, \partial_{m-1}\right) \bar{\tau}}{\bar{\tau}}=\bar{w}_{m}=O\left(z^{-1}\right)
$$

for some constant coefficient polynomial $P$, we observe by induction in $m$ that the top degree coefficient of $\bar{\tau}$ is constant in $t$, so that $\bar{\tau}$ is a monic polynomial. This proves part (1) of the theorem, and, combined with [8], proves that there is no constraints that $x_{i}(0)$ and $\xi_{i}(0)$ must satisfy. To complete the proof of part (2), we have only to determine the time evolution of $x_{i}$.

Let $w^{*}=\left(\sum w_{m}^{*} z^{-m}\right) e^{-\sum t_{i} z^{i}}, w_{0}^{*}=1$, be the adjoint Baker-Akhiezer function of $w$, i.e., $\partial_{2} w^{*}=-B_{2}^{*} w^{*}=-B_{2} w^{*}$, whose coefficients $w_{m}^{*}, m>0$, vanish at $t_{1}=\infty$. Setting

$$
w_{m+1}^{*}=\sum_{i=1}^{N} \frac{c_{m+1, i}^{*}}{t_{1}-x_{i}}, \quad m \geq 0,
$$

the same calculations as above show

$$
\mathbf{c}_{m}^{*}=(-1)^{m-1} \cdot{ }^{t} \overrightarrow{\mathbf{1}} Y^{m-1}, \quad \text { and } \quad \frac{1}{2} \partial_{2} \mathbf{c}_{m}^{*}=(-1)^{m} \cdot{ }^{t} \overrightarrow{\mathbf{1}} Y^{m-1} Z,
$$

where $\mathbf{c}_{m}^{*}=\left(c_{m, 1}^{*}, \ldots, c_{m, N}^{*}\right)$. Now we compute the coefficients of $\partial_{x}^{-1}$ in the equation

$$
\partial_{n} W=-\left(W \partial_{x}^{n} W^{-1}\right)_{-} W
$$

which is equivalent to (16). The left hand side gives

$$
\partial_{n} W=-\left(\partial_{1} \partial_{n} \log \prod\left(t_{1}-x_{i}\left(t^{\prime}\right)\right)\right) \partial_{x}^{-1}+O\left(\partial_{x}^{-2}\right)=-\sum \frac{\partial_{n} x_{i}}{\left(t_{1}-x_{i}\right)^{2}} \partial_{x}^{-1}+O\left(\partial_{x}^{-2}\right) .
$$


Comparing the formulas $w^{*}=\left(\sum w_{m}^{*}\left(-\partial_{x}\right)^{-m}\right) e^{-\sum t_{i} z^{i}}$ and $w^{*}={ }^{t} W^{-1} e^{-\sum t_{i} z^{i}}$ (see [5]), we have

$$
W^{-1}=\sum \partial_{x}^{-m} \circ w_{m}^{*},
$$

and hence the right hand side of (18) becomes

$$
\begin{aligned}
-\left(W \partial_{x}^{n} W^{-1}\right)_{-} W & =-\left(\sum_{m+m^{\prime}>n} w_{m} \partial_{x}^{n-m-m^{\prime}} \circ w_{m^{\prime}}^{*}\right)\left(1+O\left(\partial_{x}^{-2}\right)\right) \\
& =-\sum_{m+m^{\prime}=n+1} w_{m} w_{m^{\prime}}^{*} \partial^{-1}+O\left(\partial_{x}^{-2}\right) \\
& =-\sum_{\substack{m+m^{\prime}=n+1 \\
m, m^{\prime}>0}}\left(\sum_{i=1}^{N} \frac{c_{m, i} c_{m^{\prime}, i}^{*}}{\left(t_{1}-x_{i}\right)^{2}}+\text { simple poles }\right) \partial^{-1}+O\left(\partial_{x}^{-2}\right)
\end{aligned}
$$

Comparing this with (19), and using (14), (17) and $\partial Y / \partial \xi_{i}=E_{i i}$, where $E_{i j}$ denotes the $(i, j)$ th matrix element, i.e., $\left(E_{i j}\right)_{k l}=\delta_{i k} \delta_{j l}$, we have

$$
\begin{aligned}
\partial_{n} x_{i} & =\sum_{\substack{m+m^{\prime}=n+1 \\
m, m^{\prime}>0}} c_{m, i} c_{m^{\prime}, i}^{*}=\sum_{\substack{m+m^{\prime}=n+1 \\
m, m^{\prime}>0}} \mathbf{c}_{m^{\prime}}^{*} E_{i i} \mathbf{c}_{m} \\
& =(-1)^{n} \sum_{\substack{m+m^{\prime}=n+1 \\
m, m^{\prime}>0}}(1, \ldots, 1) Y^{m^{\prime}-1} E_{i i} Y^{m-1}\left(\begin{array}{c}
1 \\
\vdots \\
1
\end{array}\right) \\
& =(-1)^{n}(1, \ldots, 1) \frac{\partial Y^{n}}{\partial \xi_{i}}\left(\begin{array}{c}
1 \\
\vdots \\
1
\end{array}\right) \\
& \left.=(-1)^{n} \frac{\partial}{\partial \xi_{i}} \operatorname{tr}\left(\begin{array}{c}
1, \ldots, 1 \\
\ldots \\
\ldots \\
1, \ldots, 1
\end{array}\right)\right) \\
& =(-1)^{n} \frac{\partial}{\partial \xi_{i}} \operatorname{tr}\left(Y^{n}([X, Y]+I)\right),
\end{aligned}
$$

where $X=\operatorname{diag}\left(x_{1}, \ldots, x_{N}\right)$, and $I$ is the identity matrix. Since $\operatorname{tr}\left(Y^{n}[X, Y]\right)=$ $\operatorname{tr}\left[X, Y^{n}\right]=0,(20)$ gives the first equation in (3).

Finally, differentiating the first line of (20), and applying (14), (15), (17) and $\partial Y / \partial x_{i}=-\left[E_{i i}, Z\right]$, we have

$$
\partial_{n} \xi_{i}=\frac{1}{2} \partial_{2} \partial_{n} x_{i}=\sum_{\substack{m+m^{\prime}=n+1 \\ m, m^{\prime}>0}}\left(\mathbf{c}_{m^{\prime}}^{*} E_{i i} Z \mathbf{c}_{m}-\mathbf{c}_{m^{\prime}}^{*} Z E_{i i} \mathbf{c}_{m}\right)
$$




$$
\begin{aligned}
& =(-1)^{n} \sum_{\substack{m+m^{\prime}=n+1 \\
m, m^{\prime}>0}}(1, \ldots, 1) Y^{m^{\prime}-1}\left[E_{i i}, Z\right] Y^{m-1}\left(\begin{array}{c}
1 \\
\vdots \\
1
\end{array}\right) \\
& =-(-1)^{n}(1, \ldots, 1) \frac{\partial Y^{n}}{\partial x_{i}}\left(\begin{array}{c}
1 \\
\vdots \\
1
\end{array}\right) \\
& =-(-1)^{n} \frac{\partial}{\partial x_{i}} \operatorname{tr}\left(Y^{n}\left(\begin{array}{c}
1, \ldots, 1 \\
\ldots . \\
1, \ldots, 1
\end{array}\right)\right) \\
& =-(-1)^{n} \frac{\partial}{\partial x_{i}} \operatorname{tr} Y^{n} \quad(\text { as in the last line of }(20) \text { ), }
\end{aligned}
$$

yielding the second equation in (3).

\section{References}

[1] H. Airault, H. P. McKean, and J. K. Moser, Rational and elliptic solutions of the Korteweg-de Vries equation and a related many-body problem, Communications in Pure and Applied Mathematics, 30 (1977), 95-148.

[2] F. Calogero, Solution of the one-dimensional $N$-body problems with quadratic and/or inversely quadratic pair potentials, Journal of Mathematical Physics, 12 (1971), 419-436.

[3] — Exactly solvable one-dimensional many-body problems, Lett. Nuovo Cimento, 13 (1975), 411-416.

[4] - On a functional equation connected with integrable many-body problems, Lett. Nuovo Cimento, 16 (1976), 77-80.

[5] E. Date, M. Jimbo, M. Kashiwara, and T. Miwa, Transformation groups for soliton equations, in "Proceedings RIMS Symp. Non-linear integrable systems - classical theory and quantum theory (Kyoto 1981)", Singapore, 1983, World Scientific, 39-119.

[6] A. Kasman, To appear, 1994.

[7] I. M. Krichever, Methods of algebraic geometry in the theory of non-linear equations, Russ. Math. Surv., 32 (1977), 185-213.

[8] —, Rational solutions of Kadomtsev-Petviashvili equation and integrable systems of $n$ particles on a line, Funct. Anal. Appl., 12 (1978), 59-61. 
[9] D. Mumford, An algebro-geometric construction of commuting operators and of solutions to the Toda lattice equation, Korteweg-de Vries equation and related non-linear equations, in "Proceedings Int. Symp. Algebraic Geometry, Kyoto (1977)", Tokyo, 1978, Kinokuniya Book Store, 115-153.

[10] G. Segal and G. Wilson, Loop groups and equations of KdV type, IHES Publ. Math., 61 (1985), 5-65.

[11] T. Shiota, Characterization of Jacobian varieties in terms of soliton equations, Invent. Math., 83 (1986), 333-382.

[12] A. Treibich and J.-L. Verdier, Solitons elliptique, in "The Grothendieck Festschrift", P. Cartier et al., eds., vol. III, Birkhäuser, Boston, Basel, Stuttgart, 1990. 